\title{
Predictores del desempeño laboral de personas con discapacidad por trastorno mental severo. Revisión de estudios y análisis de evidencias.
}

\section{Variability of employment predictors for persons with severe mental illness. Studies review and research evidences}

\section{LUIS ÁNGEL LÓPEZ MENÉNDEZ*}

\begin{abstract}
RESUMEN
Este artículo presenta una revisión de la investigación sobre variables predictoras del desempeño laboral de personas con trastorno mental severo (TMS). Dentro del proceso de rehabilitación psicosocial de personas con discapacidad por TMS, la rehabilitación laboral es un elemento importante del proceso, dado que la realización de actividad laboral es un indicativo del nivel de recuperación para este colectivo. Descritas la metodologías de rehabilitación laboral, se revisan los estudios que analizan las distintas variables predictoras del desempeño laboral en personas con TMS, exponiéndose a continuación las conclusiones que de los resultados de esta revisión, se pueden extraer.
\end{abstract}

\section{PALABRAS CLAVE}

Trastorno mental severo, Rehabilitación psicosocial, Rehabilitación laboral, Predictores, Revisión.

\section{ABSTRACT}

This article presents a review of research about variability predictors of employment for persons with severe mental illness. During the process of psychosocial rehabilitation of

* PRISMA Asistencial. luis_angel_lm@hotmail.com 
persons with severe disabilities caused by severe mental illness, an important issue is the employment rehabilitation, as an indicator of the recovering lever of these persons. Once we have seen the different methodology of vocational rehabilitation, we will review the possible variability employment predictors for persons with severe mental illness, explaining as follows the review results.

\section{KEY WORDS}

Severe mental illness, Psychosocial rehabilitation, Vocational rehabilitation, Predictors, Review. 
Las personas con enfermedades mentales consideradas graves y/o crónicas, tienen alteraciones propias de su trastorno psicopatológico, además de consecuencias negativas en su integración social (funcionamiento psicosocial).

Para denominar a este colectivo se vienen usando varios términos. Con frecuencia paciente o enfermo mental crónico se utilizó con este amplio y heterogéneo colectivo, que tienen en común padecer enfermedades mentales severas de larga duración; con el paso del tiempo se elimina el uso del término crónico, pues no se corresponde con la realidad del colectivo (Ciompi, 1980; Shepherd, Watt, Falloon y Smeeton, 1989), además de suscitar juicios negativos y estigmatizantes. Vocablos como enfermedad mental grave y duradera (IMSERSO, 2007a) o trastorno mental grave y persistente (IMSERSO, 2007b) son también utilizados. El término trastorno mental severo (TMS) cuenta con un significativo consenso científico, para referirse a los trastornos mentales graves de duración prolongada y que conllevan un grado variable de discapacidad y disfunción social (Gisbert, 2002).

Se considera TMS cuando además del diagnóstico, que generalmente incluye esquizofrenia, otros trastornos psicóticos, desorden bipolar, depresión mayor y trastornos de la personalidad (Mueser, Bond y Drake, 2001), concurre una duración prolongada del trastorno, que en muchos casos es de carácter crónico, además de la presencia de discapacidad con una evidente dificultad para el desenvolvimiento autónomo en la vida personal y social (NIMH, 1987).

Este colectivo tiene una significativa disfunción sociolaboral, dado su bajo índice de empleo competitivo (empleo normalizado), que según los estudios es del 5\% al 20\% (Anthony y Blanch, 1987;
FEAFES, 1995; Lauriello, Lenroot y Bustillo, 2003). A pesar de las consideraciones previas que daban escasa importancia al diagnóstico para predecir las posibilidades de empleo de las personas con TMS (Anthony y Blanch, 1987; Anthony, 1994), estudios recientes indican que las personas con diagnóstico de esquizofrenia consiguen significativamente menos empleos que otras con otro diagnóstico propio de los TMS (Cook y Razzano, 2000); en torno al 90\% no tienen empleo (López, Alvarez y Laviana, 1998; Mueser, Salyers y Mueser, 2001; Perkins y Rinaldi, 2002) y la mayoría de los que lo obtienen suelen perderlo en un plazo medio de seis meses (Resnick y Bond, 2001). Este es el grupo que presenta mayor complejidad, a la hora de plantearse maneras efectivas de ayudar a sus miembros a estar y mantenerse laboralmente activos (Lehman, 1995; Penn y Mueser, 1996; Boardman, Grove, Perkins y Shepherd, 2003).

De las personas que por primera vez ingresan en unidades de salud mental, el $53 \%$ de los pacientes que reciben diagnóstico de esquizofrenia tenían empleo antes de la hospitalización, siendo este porcentaje del $66 \%$ en el trastorno bipolar y de $75 \%$ en otros trastornos psicóticos (Muntaner y O'Campo, 1993).

Respecto del sexo, un $17,1 \%$ de las mujeres con esquizofrenia tienen trabajo remunerado a tiempo completo, mientras que esta situación laboral sólo se da en un 5,7\% de hombres con ese mismo diagnóstico (Andia, Zisook, Heaton, Hesselink y Jernigan, 1995; Jiménez, Vázquez y Waisman, 2000).

Numerosas dificultades, que resultan del encadenamiento de distintos factores, entorpecen la integración laboral de las personas con esquizofrenia:

- La enfermedad y su sintomatología 
afecta áreas funcionales de la persona, como la cognitiva, perceptiva, afectiva y relacional (Wing, 1992; Ancil, Holliday y Higgenbttam, 1994; Aldaz y Vázquez, 1996).

- Escasa formación académica y profesional (McGurrin, 1994).

- Efectos secundarios de la medicación y procesos de rehabilitación inadecuados (Wing, 1992; Anthony, 1994; López y Laviana, 1998).

- Barreras sociales, como el desconocimiento que la sociedad tiene hacia este colectivo (Rosen y Barfoot, 2001), las ideas prejuiciadas, el estigma social (Simmons, Selleck, Steele y Sepetauc, 1993; Manning y Withe, 1995; Saraceno, 1995; Perkins y Rinaldi, 2002) y el efecto desmotivador hacia la actividad laboral de las pensiones (López, Alvarez y Laviana, 1998; Turton, 2001; Warner, 2001).

A pesar de estas dificultades, según se acredita en un amplio número de estudios, la integración laboral de las personas con TMS es posible, (Anthony y Blanch, 1987; Bond, 1994; Bond, Drake, Mueser y Becker, 1997; Cook y Razzano, 2000; Bond, Becker, Drake, Rapp, Meisler y Lehman, 2001; Crowther, Marshall, Bond y Huxley, 2001; O'Flynn y Craig, 2001; Boardman, Grove, Perkins y Shepherd, 2003; Bond, 2003; Shepherd, 2003).

La mayoría de las personas con TMS desean tener un empleo competitivo (Rogers, Walsh, Masotta y Danley 1991; Bebout y Harris, 1995; Mueser, Salyers y Mueser, 2001) y remunerado (Anthony, Cohen y Farkas, 1990). A pesar del general interés por incorporarse a la actividad laboral, un significativo porcentaje de afectados se autovaloran con poca capacidad para el desempeño en la actividad laboral (Hays y Buckle, 1992; Lecomte, Cyr, Lesage, Wilde, Leclerc y Ricard, 1999; Roe, 2003).

La actividad laboral tiene una serie de funciones:

- Productiva: potenciador de desarrollo cognitivo, organizador de la vida cotidiana y facilitador de relaciones sociales (Mueser y Tarrier, 1998; Rosen y Barfoot, 2001).

- Retributiva: permite la autonomía económica necesaria para desempenar un rol social activo (Bell, Lysaker y Milstein, 1996; Bryson, Lysaker y Bell, 2002).

- Valoración social: aumenta la autoestima al tener un rol de trabajador y el reconocimiento social que esto implica (Shepherd, 1989; Farina, 1998; Torrey, Mueser, McHugo y Drake, 2000; Perkins y Rinaldi, 2002; Goergen, 2003), siendo por tanto el estigma menor en personas con TMS empleados, que en los no empleados (Link, Cullen, Mirotznik y Struening, 1992).

La actividad laboral, para las personas con TMS, es tanto resultado como determinante de su evolución (Liberman, 1993). No tiene un claro efecto sobre la sintomatología positiva, no es antecedente de mejorias clínicas ni descompensaciones significativas; tampoco es esperable un gran efecto sobre la sintomatología negativa, en relación a la cual se observan ligeras mejorias. Por el contrario se constata un positivo efecto sobre el funcionamiento social, el grado de satisfacción, la autonomía personal, la autoestima, y en general, sobre la calidad de vida de las personas con TMS (Bell, Lysaker y Milstein, 1996; Hayes y Halford, 1996; Holzner, Kemmler y Meise, 
1998; Casper y Fishbein, 2000; Mueser, Becker y Wolfe, 2001; Resnick y Bond, 2001; Bryson, Lysaker y Bell, 2002).

Aguado (1995) hace un exhaustivo estudio de la historia de las deficiencias, aportando amplia información respecto de las personas con trastorno mental severo; revisa las distintas fases que la atención de este colectivo ha tenido. La forma de entender y atender a las personas con diagnóstico de enfermedad mental ha ido cambiando (Posininsky y Schaumburg, 1996), siendo habitual el uso de tratamientos donde se propician actividades ocupacionales, incluso de carácter productivo.

La Asamblea General de la ONU en su resolución 46/119, de 17 de diciembre de 1991, establece los principios para la protección de los enfermos mentales y el mejoramiento de la atención de la salud mental. En el principio 3 se indica que toda persona que padezca una enfermedad mental tendrá derecho a vivir y a trabajar, en la medida de lo posible, en la comunidad; el principio 9 señala que el tratamiento de cada paciente estará destinado a preservar y estimular su independencia personal.

Este modelo de atención basado en la asistencia en la comunidad (Desviat, 1995; Gisbert, 2002) se propone evitar la institucionalización. En España se han realizado notables avances en los servicios de salud mental, precisándose potenciar recursos de rehabilitación y soporte social (redes de cuidados intermedios y sociosanitarios), especialmente en cuanto se refiere a las alternativas residenciales y laborales para personas con TMS con discapacidad o minusvalía (Defensor del Pueblo, 1992).

La asistencia rehabilitadora para personas con discapacidad empezó con los discapacitados físicos interesándose por los déficit de carácter somático, para pronto pasar a abordar los aspectos psicosociales y muy en especial la recuperación del empleo (Aguado y Alcedo, 1990), tomando poco a poco la rehabilitación laboral una importante presencia en la atención de las personas con discapacidad.

\section{REHABILITACIÓN LABORAL}

Ciardiello y Bell (1988) definen la rehabilitación laboral como un proceso específico, estructurado e individualizado dirigido a ayudar a preparar a las personas con TMS para que accedan y se mantengan en el mundo laboral. Conlleva intervenciones complejas e individualizadas, con atenciones variadas y coordinadas aportadas por diversos servicios asistenciales y que pueden los usuarios necesitarlas durante largo tiempo (Peckham y Muller, 1999; Becker y Smith, 2001; Furlong, McCoy, Dincin, Clay, McClory y Pavick, 2002; Lal y Mercier, 2002; Boardman, Grove, Perkins y Shepherd, 2003; Kravetz, Dellario, Granger y Salzer, 2003).

No hay factores que aconsejen la exclusión del acceso al empleo de una persona con TMS (Becker y Smith, 2001); la integración laboral se consigue cuando se cumplen las siguientes condiciones (Danley, 1993):

- Ámbito laboral donde los trabajadores mayoritariamente no son discapacitados.

- Al menos 20 horas de trabajo a la semana.

- Sueldo homologable al de otro trabajador no discapacitado, con iguales condiciones laborales.

- Apoyo mínimo en el lugar de trabajo, el cual se reduce progresivamente. 
La rehabilitación laboral de personas con TMS ha ido desarrollando programas asistenciales, que han ido avanzando de los primeros objetivos ocupacionales a los de inserción laboral y por fin de integración laboral normalizada. Las primeras iniciativas fueron de laborterapia, realizada sobre todo en instituciones psiquiátricas, buscando que los usuarios mantengan un nivel de ocupación programada dentro del recinto de la institución. La implantación del modelo de atención comunitaria para este colectivo, ha impulsado acciones de facilitación del desempeño laboral en entornos comunitarios.

Los modelos de rehabilitación laboral hoy en día más utilizados son el Empleo con Apoyo (Supported Employment Approach - SEA) y el Empleo Protegido (EP).

SEA es un modelo de integración laboral para personas con discapacidad grave, que comenzó a desarrollarse en Estados Unidos en los años 80 (Wehman, 1981; Wehman, Moon, Everson, Wood y Barcus, 1987); la psicología experimental y la modificación de conducta fueron las fuentes de influencia más importantes para el nacimiento del SEA (Wehman, 1981; Snell 1983). Es aplicado a personas con TMS a partir de los años 90 en Estados Unidos por Bond (Bond, 1992; Bond, 1994; Bond, Drake, Mueser y Becker, 1997; Bond, 1998; Bond, Drake, Becker y Mueser, 1999; Bond, Becker, Drake, Rapp, Meisler y Lehman, 2001) y en Canadá por Latimer (1999), con el objetivo de ser una alternativa eficaz a los métodos tradicionales de rehabilitación laboral para este colectivo. Factores clave del SEA:

- Tenaz búsqueda y mantenimiento de empleo ordinario.

- Apoyos individualizados, flexibles y continuados en el tiempo, con espe- cial referencia a los derivados de la interacción cotidiana con otros trabajadores de su ámbito laboral (Marrone, Balzell y Gold, 1995; Drake, McHugo, Bebout, Becker, Harris, Bond y Quimby, 1999; Banks, Charleston, Grossi y Mank, 2001; Henry, Barreira, Banks, Brown y McKay, 2001; Dorio, Guitar, Solheim, Dvorkin y Marine, 2002; Furlong, McCoy, Dincin, Clay, McClory y Pavick, 2002; McDonaldWilson, Rogers, Massaro, Lyass y Crean, 2002; Gowdy, Carlson y Rapp, 2003).

- Relativa utilidad de la formación previa, realizándose la formación y el seguimiento en el propio puesto de trabajo (Wallace, Tauber y Wilde, 1999).

- Trabajo normalizado e interacción cotidiana con personas no enfermas, con reglas de funcionamiento comunes (Banks, Charleston, Grossi y Mank, 2001; Furlong, McCoy, Dincin, Clay, McClory y Pavick, 2002; McDonald-Wilson, Rogers, Massaro, Lyass y Crean, 2002; Gowdy, Carlson y Rapp, 2003).

SEA tiene dos variantes:

1. Actividad laboral desde el inicio con disponibilidad de servicios de apoyo (Cook y Razzano, 2000):

- Actividad laboral en el puesto de trabajo.

- Entrenamiento laboral desde el inicio.

- Disponibilidad, de forma continua, tanto de entrenamiento como de apoyo vocacional, realizados por "facilitadores".

- Remuneración. 
2. Entrenamiento inicial y posterior actividad laboral.

La primera de estas opciones del SEA ha demostrado ser más eficaz (Bustillo, Lauriello, Horan y Keith, 2001).

Los usuarios principales de los programas de SEA en España siguen siendo las personas con discapacidad por retraso mental, posteriormente y en igual proporción las personas con discapacidad fisica o sensorial, y finalmente con escasa representación en las personas con discapacidad por enfermedad mental (Verdugo, Jordán de Urríes y Bellver, 1998; Verdugo y Jordán de Urries, 2003).

El empleo con apoyo es un eficaz método de integración laboral (Verdugo y Jordán de Urríes, 2003), siendo evaluado en numerosos estudios (Gervey y Bedell, 1993; Bond, 1994; Bond, Dietzen, McGrew y Miller, 1995; Drake, McHugo, Becker, Anthony y Clark, 1996; Penn y Mueser, 1996; Bond, Drake, Mueser y Becker, 1997; Chandler, Meisel, Hu, McGowen y Madison, 1997; Bond, 1998; Drake, McHugo, Bebout, Becker, Harris, Bond y Quimby, 1999; Cook y Razzano, 2000; McFarlane, Dushay, Deakins, Stastny, Lukens, Toran y Link, 2000; Bond, Becker, Drake, Rapp, Meisler y Lehman, 2001; Crowther, Marshall, Bond y Huxley, 2001; Lehman, Goldberg, Dixon, McNary, Postrado, Hackman y McDonnell, 2002; Bond y Drake, 2008); es más efectivo que los métodos vocacionales tradicionales (Burns, Catty, White, Becker, Koletsi, Fioritti, Rössler, Tomov, Van Busschbach, Wiersma y Lauber, 2008; Catty, Lissouba, White, Becker, Drake, Fioritti, Knapp, Lauber, Rössler, Tomov, Van Busschbach, Wiersma, y Burns, 2008); los resultados de las investigaciones indican:

- Un promedio del 58\% de usuarios de SEA obtenían trabajo y siendo del
$21 \%$ en usuarios de acercamientos vocacionales tradicionales.

- Los usuarios de programas de SEA trabajaron más horas y obtuvieron salarios más elevados.

SEA en coordinación con servicios psiquiátricos y vocacionales, es más eficaz que con ausencia de esos servicios (Cook, Lehman, Drake, McFarlane, Gold, Leff, Blyler, Toprac, Razzano, BurkeMiller, Blankertz, Shafer, Pickett-Schenk y Grey, 2005).

El EP se utiliza en la mayoría de países europeos como mecanismo de inserción de las personas con discapacidad en el mercado laboral (Laloma, 2007). Las empresas generadoras de EP en España son los Centros Especiales de Empleo (CEE), que son empresas que adquieren esta catalogación y han de contar con un porcentaje de trabajadores con discapacidad no inferior al $70 \%$ de su plantilla. Los Enclaves Laborales es también una fórmula de EP que pretende acercar a los empleados de los CEE a las empresas ordinarias. Numerosos estudios indican que los CEE favorecen el acceso y mantenimiento en el empleo a personas con discapacidad (Gallio, 1987; Saraceno, 1995; Grove, Freudenberg, Harding y O’Flynn, 1997; López, Alvarez y Laviana, 1998; Boardman, Grove, Perkins, Perkins y Shepherd, 2003; Bond, 2003; Goergen, 2003; Schwarz, 2003; Shepherd, 2003).

\section{PREDICTORES DEL DESEMPEÑO LABORAL}

La determinación de variables predictoras es de gran importancia en todo tipo intervenciones asistenciales; en la rehabilitación laboral de personas con TMS, una de las dificultades de los profesionales de esta disciplina, es decidir qué 
usuario es susceptible de realizar con buenos resultados el proceso rehabilitador y cuál no (Sobrino, 2003).

El estudio de variables predictoras de éxito o fracaso de la rehabilitación laboral de personas con TMS, ha generado numerosos trabajos de investigación, los cuales dada la heterogeneidad de las poblaciones estudiadas, los diferentes programas de rehabilitación laboral considerados y las variadas metodologias de investigación, de medición de variables y de evaluación de resultados, hace inviable sacar conclusiones definitivas, aunque sí se pueden señalar resultados, que por su solidez, son de tener en cuenta. Los investigadores examinan sobre todo predictores de tres tipos: características de los pacientes, los factores ambientales y las intervenciones (Bond y Drake, 2008).
Orviz y González (1994) realizaron una interesante revisión de estudios disponibles sobre predictores de éxito o fracaso en la integración laboral de personas con TMS, con los resultados que se detallan en el cuadro 1.

Con población española con discapacidad por enfermedad mental, Caleidoscopia Investigación Social (1997) realiza un estudio en el que se recogen las consideraciones de los profesionales de servicios de rehabilitación laboral, respecto de los predictores de integración laboral para este colectivo, cuyos resultados se indican en el cuadro 2.

En los últimos años se han seguido realizando numerosas investigaciones para el estudio de las variables que predicen el desempeño laboral de las perso-

\section{Cuadro 1}

\begin{tabular}{|l|l|}
\hline Si predicen & No predicen \\
\hline - Tener habilidades de ajuste laboral, evaluadas & - El diagnóstico psiquiátrico. \\
en contextos laborales. & - Historia de la enfermedad. \\
- Historia laboral previa. & - Resultados de test de inteligencia. \\
- Expectativas e intereses ajustados. & - Funcionamiento en ambientes no laborales. \\
- Autoestima de la persona como trabajador. & - Sintomatología psicopatológica presente. \\
- Exito en programas de formación para & \\
el empleo. & \\
- Disponer de apoyo profesional durante largo & \\
plazo una vez que realiza actividades laborales. & \\
- Motivación hacia la incorporación laboral. & \\
\hline
\end{tabular}

\section{Cuadro 2}

\section{Factores necesarios (imprescindibles):}

- Apoyo familiar.

- Capacidad de adaptación a situaciones de tensión e inestabilidad.

Factores favorecedores (prescindibles):

- Compensado psicopatológicamente.

- Entorno familiar estable.

- Conciencia de enfermedad.

- Motivación laboral.

- Alta autoestima.

Factores negativos (entorpecedores):

- Escasa experiencia laboral.

- Baja cualificación.

- Tener ayuda económica (pensión). 
nas con TMS, con los resultados que se indican en los cuadros siguientes.

Las experiencias laborales previas, el grado de éxito de las mismas y el nivel de formación, son predictores de la inserción laboral (Anthony, 1994; Anthony, Rogers, Cohen y Davies, 1995; Rogers, Anthony, Cohen y Davies, 1997; Mueser, Bond y Drake, 2001; Nordt, Müller, Rössler y Lauber, 2007; Catty, Lissouba, White, Becker, Drake, Fioritti, Knapp, Lauber, Rössler, Tomov, Van Busschbach, Wiersma, y Burns, 2008).

Bajo nivel de capacidad funcional premórbido, están asociados a desempleo en personas con esquizofrenia (McGurk y Meltzer, 2000).

Pocas hospitalizaciones psiquiátricas y alto grado de formación, son predictores de mayor nivel de integración laboral (Nordt, Müller, Rössler y Lauber, 2007).

Los episodios de hospitalización psiquiátrica (número y duración) no es factor predictor de integración laboral (Anthony, 1994).

El trabajo en una actividad de preferencia, es mantenido el doble de tiempo que aquellos empleos en otras actividades. Mayor satisfacción con el trabajo, cuando este se realiza en una actividad de preferencia (Becker y Smith, 2001).

Tener deseo de trabajar, tiene una influencia positiva en el funcionamiento en los programas de rehabilitación laboral (Bond, 1994; Lysaker, Bell, Zito y Bioty, 1995; Cook y Razzano, 2000; Warner, 2001; Provencher, Gregg, Mead y Mueser, 2002).

La creencia negativa sobre uno mismo, es predictor de un resultado más pobre de desempeño laboral y de tener una actitud evasiva respecto de la actividad laboral (Van Dongen, 1998; Regenold, Sherman y Fenzel, 1999; Hoffmann, Kupper y Kunz, 2000; Lysaker, Clements, Wright, Evans y Marks, 2001).

Tener expectativas altas de éxito no se relaciona con mejor funcionamiento laboral; expectativa de éxito laboral baja o moderada, correlaciona con mejores resultados laborales (Davis, Nees, Hunter y Lysaker, 2004).

Las expectativas de desempeño laboral son en general ajustadas a sus posibilidades laborales (Bond, Drake y Bec-

\section{Cuadro 3. Análisis del valor predictor de la situación premórbida}

Las experiencias laborales previas, el grado de éxito de las mismas y el nivel de formación, son predictores de la inserción laboral (Anthony, 1994; Anthony, Rogers, Cohen y Davies, 1995; Rogers, Anthony, Cohen y Davies, 1997; Mueser, Bond y Drake, 2001; Nordt, Müller, Rössler y Lauber, 2007; Catty, Lissouba, White, Becker, Drake, Fioritti, Knapp, Lauber, Rössler, Tomov, Van Busschbach, Wiersma, y Burns, 2008).

Bajo nivel de capacidad funcional premórbido, están asociados a desempleo en personas con esquizofrenia (McGurk y Meltzer, 2000).

Pocas hospitalizaciones psiquiátricas y alto grado de formación, son predictores de mayor nivel de integración laboral (Nordt, Müller, Rössler y Lauber, 2007).

Los episodios de hospitalización psiquiátrica (número y duración) no es factor predictor de integración laboral (Anthony, 1994). 


\section{Cuadro 4. Análisis del valor predictor de los factores actitudinales}

El trabajo en una actividad de preferencia, es mantenido el doble de tiempo que aquellos empleos en otras actividades. Mayor satisfacción con el trabajo, cuando este se realiza en una actividad de preferencia (Becker y Smith, 2001).

Tener deseo de trabajar, tiene una influencia positiva en el funcionamiento en los programas de rehabilitación laboral (Bond, 1994; Lysaker, Bell, Zito y Bioty, 1995; Cook y Razzano, 2000; Warner, 2001; Provencher, Gregg, Mead y Mueser, 2002).

La creencia negativa sobre uno mismo, es predictor de un resultado más pobre de desempeño laboral y de tener una actitud evasiva respecto de la actividad laboral (Van Dongen, 1998; Regenold, Sherman y Fenzel, 1999; Hoffmann, Kupper y Kunz, 2000; Lysaker, Clements, Wright, Evans y Marks, 2001).

Tener expectativas altas de éxito no se relaciona con mejor funcionamiento laboral; expectativa de éxito laboral baja o moderada, correlaciona con mejores resultados laborales (Davis, Nees, Hunter y Lysaker, 2004).

Las expectativas de desempeño laboral son en general ajustadas a sus posibilidades laborales (Bond, Drake y Becker, 1998; Resnick y Bond, 2001; Provencher, Gregg, Mead y Mueser, 2002).

ker, 1998; Resnick y Bond, 2001; Provencher, Gregg, Mead y Mueser, 2002).

Altos niveles de sintomatología en personas con esquizofrenia y otros trastornos psicóticos, se asocian a desempeño laboral pobre, en lo relativo a las habilidades laborales y a menor probabilidad de obtener un empleo remunerado a tiempo completo (Anthony, Rogers, Cohen y Davis, 1995; Salkever, Karakus, Slade, Harding, Hough, Rosenheck, Swartz, Barrio y Yamada, 2007).

Los sintomas positivos y negativos de la esquizofrenia predicen los resultados del empleo (Beiser, Bean, Erickson, Iacono y Rector, 1994; Daradkeh y Karim, 1994).

Los síntomas negativos sí predicen los resultados del empleo; no lo hacen los sintomas positivos (Mueser, Salyers, Mueser, 2001; Slade y Salkever, 2001; Mueser, 2002).
Los síntomas negativos persistentes, son predictores de inhabilidad social y laboral (Bailer, Bräuer, Rey, 1996; McGurk y Meltzer, 2000; Milev, Ho, Arndt y Andreasen, 2005).

Sintomas negativos y hospitalizaciones recientes, se asocian con bajos logros laborales (Razzano, Cook, BurkeMiller, Mueser, Pickett-Schenk, Grey, Goldberg, Blyler, Gold, Leff, Lehman, Shafer, Blankertz, McFarlane, Toprac y Carey, 2005).

El efecto sobre el empleo de una reducción de los sintomas negativos, es significativamente mayor que el efecto de una reducción comparable de los sintomas positivos; este efecto es menos significativo en el empleo con apoyo, que en otros modelos de rehabilitación laboral (Slade y Salkever, 2001).

Es mayor la dificultad laboral cuando 


\section{Cuadro 5. Análisis del valor predictor de la sintomatologia}

Altos niveles de sintomatología en personas con esquizofrenia y otros trastornos psicóticos, se asocian a desempeño laboral pobre, en lo relativo a las habilidades laborales y a menor probabilidad de obtener un empleo remunerado a tiempo completo (Anthony, Rogers, Cohen y Davis, 1995; Salkever, Karakus, Slade, Harding, Hough, Rosenheck, Swartz, Barrio y Yamada, 2007).

Los sintomas positivos y negativos de la esquizofrenia predicen los resultados del empleo (Beiser, Bean, Erickson, Iacono y Rector, 1994; Daradkeh y Karim, 1994).

Los sintomas negativos sí predicen los resultados del empleo; no lo hacen los sintomas positivos (Mueser, Salyers, Mueser, 2001; Slade y Salkever, 2001; Mueser, 2002).

Los sintomas negativos persistentes, son predictores de inhabilidad social y laboral (Bailer, Bräuer, Rey, 1996; McGurk y Meltzer, 2000; Milev, Ho, Arndt y Andreasen, 2005).

Sintomas negativos y hospitalizaciones recientes, se asocian con bajos logros laborales (Razzano, Cook, Burke-Miller, Mueser, Pickett-Schenk, Grey, Goldberg, Blyler, Gold, Leff, Lehman, Shafer, Blankertz, McFarlane, Toprac y Carey, 2005).

El efecto sobre el empleo de una reducción de los síntomas negativos, es significativamente mayor que el efecto de una reducción comparable de los sintomas positivos; este efecto es menos significativo en el empleo con apoyo, que en otros modelos de rehabilitación laboral (Slade y Salkever, 2001).

Es mayor la dificultad laboral cuando hay una significativa sintomatologia negativa y escasas habilidades sociales (Lysaker y Bell, 1995; Lysaker, Bell, Zito y Bioty, 1995; Rogers, Anthony, Cohen y Davies, 1997; Suslow, Schonauer, Ohrmann, Eikelmann y Reker, 2000).

Son valorados como más empleables, quienes tienen mayores habilidades comunicacionales y mayor ajuste social. Son valorados como menos empleables, quienes tienen altas puntuaciones en sintomas negativos y menos habilidades durante la entrevista (Tsang, Lam, Nig y Leung, 2000).

Las habilidades sociales comunicaciones predicen el funcionamiento vocacional, con independencia del funcionamiento cognoscitivo (Dickinson, Bellack y Gold, 2006).

No hay relación entre sintomatología y resultados del empleo (Racenstein, Harrow, Reed, Martin, Herbener y Penn, 2002).

hay una significativa sintomatología negativa y escasas habilidades sociales (Lysaker y Bell, 1995; Lysaker, Bell, Zito y Bioty, 1995; Rogers, Anthony, Cohen y Davies, 1997; Suslow, Schonauer, Ohrmann, Eikelmann y Reker, 2000).

Son valorados como más empleables, quienes tienen mayores habilidades comunicacionales y mayor ajuste social. Son valorados como menos empleables, quienes tienen altas puntuaciones en síntomas negativos y menos habilidades durante la entrevista (Tsang, Lam, Nig y Leung, 2000).

Las habilidades sociales comunicaciones predicen el funcionamiento vocacio- 
nal, con independencia del funcionamiento cognoscitivo (Dickinson, Bellack y Gold, 2006).

No hay relación entre sintomatología y resultados del empleo (Racenstein, Harrow, Reed, Martin, Herbener y Penn, 2002).

Mayores dificultades para la integración laboral en las personas con esquizofrenia que las de otros tipos de personas con TMS (Anthony, Rogers, Cohen y Davies, 1995; Lysaker, Bell, Zito y Bioty, 1995; Cook y Razzano, 2000).

Déficit en el funcionamiento cognitivo dificulta la actividad laboral (Lysaker y Bell, 1995; Lysaker, Bell, Zito y Bioty, 1995; Green, 1996; Brekke, Raine, Ansel, Lencz y Bird, 1997; Hoffman y Kupper, 1997; Mueser, Becker, Torrey, Xie, Bond, Drake y Dain, 1997; Rogers, Anthony, Cohen y Davies, 1997; Bryson, Bell, Kaplan y Greig, 1998; Bellack, Gold y Buchanan, 1999; Gold, Queern, Iannone y Buchanan, 1999; McGurk y Meltzer, 2000; Suslow, Schonauer, Ohrmann, Eikelmann y Reker, 2000; Velligan, Bow-Thomas, Miller y Halgunseth, 2000; Bell y Bryson, 2001; Goldberg, Lucksted, McNary, Gold, Dixon y Lehman, 2001; Mueser, Salyers y Mueser, 2001; Gold, Goldberg, McNary, Dixon y Lehman, 2002; McGurk, Mueser, Harvey, LaPuglia y Marder, 2003; Holthausen, Wiersma, Cahn, Kahn, Dingemans, Schene y Van den Bosch, 2007).

El potencial de aprendizaje de la persona con esquizofrenia, valorado mediante el test de Wisconsin (Heaton, Chelune, Talley, Kay y Curtiss, 1993), predice su capacidad de adquisición de habilidades laborales y de desempeño laboral (Kern, Liberman, Kopelowicz, Mintz y Green, 2002; Sergi, Kern, Mintz y Green, 2005).
La capacidad de aprendizaje, la memoria verbal y la desorganización cognitiva, son predictores significativos de la conducta laboral (Evans, Bond, Meyer, Kim, Lysaker, Gibson y Tunis, 2004).

Mejoras en memoria verbal a corto y a largo plazo predicen mejor la actividad laboral, que la historia laboral previa (Vauth, Corrigan, Clauss, Dietl, DreherRudolph, Stieglitz y Vater, 2005).

El funcionamiento ejecutivo se relaciona con el desempeño funcional en las personas con esquizofrenia, en especial con el desempeño laboral (Green, Kern, Braff y Mintz, 2000; McGurk y Meltzer, 2000).

El funcionamiento cognoscitivo es predictor significativo del mantenimiento del trabajo, pero no de la obtención del mismo (Gold, Goldberg, McNary, Dixon y Lehman, 2002).

La capacidad de atención es profética del funcionamiento laboral (Milev, Ho, Arndt y Andreasen, 2005).

El funcionamiento cognoscitivo es un factor más predictivo de la actividad laboral, que la sintomatología; su capacidad predictiva es mayor a medio plazo (3-4 años) que a corto plazo (1-2 años). La capacidad de aprendizaje, la memoria $\mathrm{y}$ las funciones ejecutivas se relacionan con el desempeño laboral (McGurk y Mueser, 2006).

Sintomas cognoscitivos no se relacionan con la actividad laboral (Slade y Salkever, 2001).

No hay datos concluyentes sobre la relación entre las variables socio-demográficas e integración laboral (Lysaker, Bell, Zito y Bioty, 1995; Rogers, Anthony, Cohen y Davies, 1997; Jones, Perkins y Born, 2001; Bond y Drake, 2008). 


\section{Cuadro 6. Análisis del valor predictor del diagnóstico}

Mayores dificultades para la integración laboral en las personas con esquizofrenia que las de otros tipos de personas con TMS (Anthony, Rogers, Cohen y Davies, 1995; Lysaker, Bell, Zito y Bioty, 1995; Cook y Razzano, 2000).

\section{Cuadro 7. Análisis del valor predictor del funcionamiento cognitivo}

Déficit en el funcionamiento cognitivo dificulta la actividad laboral (Lysaker y Bell, 1995; Lysaker, Bell, Zito y Bioty, 1995; Green, 1996; Brekke, Raine, Ansel, Lencz y Bird, 1997; Hoffman y Kupper, 1997; Mueser, Becker, Torrey, Xie, Bond, Drake y Dain, 1997; Rogers, Anthony, Cohen y Davies, 1997; Bryson, Bell, Kaplan y Greig, 1998; Bellack, Gold y Buchanan, 1999; Gold, Queern, Iannone y Buchanan, 1999; McGurk y Meltzer, 2000; Suslow, Schonauer, Ohrmann, Eikelmann y Reker, 2000; Velligan, Bow-Thomas, Miller y Halgunseth, 2000; Bell y Bryson, 2001; Goldberg, Lucksted, McNary, Gold, Dixon y Lehman, 2001; Mueser, Salyers y Mueser, 2001; Gold, Goldberg, McNary, Dixon y Lehman, 2002; McGurk, Mueser, Harvey, LaPuglia y Marder, 2003; Holthausen, Wiersma, Cahn, Kahn, Dingemans, Schene y Van den Bosch, 2007).

El potencial de aprendizaje de la persona con esquizofrenia, valorado mediante el test de Wisconsin (Heaton, Chelune, Talley, Kay y Curtiss, 1993), predice su capacidad de adquisición de habilidades laborales y de desempeño laboral (Kern, Liberman, Kopelowicz, Mintz y Green, 2002; Sergi, Kern, Mintz y Green, 2005).

La capacidad de aprendizaje, la memoria verbal y la desorganización cognitiva, son predictores significativos de la conducta laboral (Evans, Bond, Meyer, Kim, Lysaker, Gibson y Tunis, 2004).

Mejoras en memoria verbal a corto y a largo plazo predicen mejor la actividad laboral, que la historia laboral previa (Vauth, Corrigan, Clauss, Dietl, Dreher-Rudolph, Stieglitz y Vater, 2005).

El funcionamiento ejecutivo se relaciona con el desempeño funcional en las personas con esquizofrenia, en especial con el desempeño laboral (Green, Kern, Braff y Mintz, 2000; McGurk y Meltzer, 2000).

El funcionamiento cognoscitivo es predictor significativo del mantenimiento del trabajo, pero no de la obtención del mismo (Gold, Goldberg, McNary, Dixon y Lehman, 2002).

La capacidad de atención es profética del funcionamiento laboral (Milev, Ho, Arndt y Andreasen, 2005).

El funcionamiento cognoscitivo es un factor más predictivo de la actividad laboral, que la sintomatologia; su capacidad predictiva es mayor a medio plazo (3-4 años) que a corto plazo (1-2 años). La capacidad de aprendizaje, la memoria y las funciones ejecutivas se relacionan con el desempeño laboral (McGurk y Mueser, 2006).

Sintomas cognoscitivos no se relacionan con la actividad laboral (Slade y Salkever, 2001). 


\section{Cuadro 8. Análisis del valor predictor del ámbito social}

No hay datos concluyentes sobre la relación entre las variables socio-demográficas e integración laboral (Lysaker, Bell, Zito y Bioty, 1995; Rogers, Anthony, Cohen y Davies, 1997; Jones, Perkins y Born, 2001; Bond y Drake, 2008).

Factores como las ayudas por incapacidad o la raza, influyen en la participación en el empleo normalizado, el cual se facilita con la participación en servicios de rehabilitación (Rosenheck, Leslie, Keefe, McEvoy, Swartz, Perkins, Stroup, Hsiao y Lieberman, 2006).

Las tasas de desempleo local tienen poco efecto sobre la integración laboral (Salkever, Karakus, Slade, Harding, Hough, Rosenheck, Swartz, Barrio y Yamada, 2007).

\section{Cuadro 9: Análisis del valor predictor del tratamiento farmacológico}

Se observa un índice más alto de empleo en aquellos pacientes que utilizan antipsicóticos de segunda generación, que aquellos que toman antipsicóticos de primera generación (McGurk y Meltzer, 2000).

No se identifican diferencias en el desempeño laboral en pacientes que usan antipsicóticos de primera o de segunda generación (Meyer, Bond, Tunis y McCoy, 2002; Bond, Kim, Meyer, Gibson, Tunis, Evans, Lysaker, McCoy, Dincin y Xie, 2004).

Factores como las ayudas por incapacidad o la raza, influyen en la participación en el empleo normalizado, el cual se facilita con la participación en servicios de rehabilitación (Rosenheck, Leslie, Keefe, McEvoy, Swartz, Perkins, Stroup, Hsiao y Lieberman, 2006).

Las tasas de desempleo local tienen poco efecto sobre la integración laboral (Salkever, Karakus, Slade, Harding, Hough, Rosenheck, Swartz, Barrio y Yamada, 2007).

Se observa un índice más alto de empleo en aquellos pacientes que utilizan antipsicóticos de segunda generación, que aquellos que toman antipsicóticos de primera generación (McGurk y Meltzer, 2000).

No se identifican diferencias en el desempeño laboral en pacientes que usan antipsicóticos de primera o de segunda generación (Meyer, Bond, Tunis y McCoy,
2002; Bond, Kim, Meyer, Gibson, Tunis, Evans, Lysaker, McCoy, Dincin y Xie, 2004).

\section{CONCLUSIONES}

Las personas con TMS, y sobre todo aquellas con diagnóstico de esquizofrenia, tienen gran dificultad para tener actividad laboral; dificultades estas de devienen a consecuencia de sus propias alteraciones psicopatológicas y los tratamientos que reciben, como también del estigma que la sociedad mantiene hacia este colectivo. Tener empleo es frecuente objetivo común de las personas con TMS, siendo la actividad laboral resultado y determinante de su evolución.

La rehabilitación laboral sigue, con este colectivo, el paso del modelo asistencial predominante en cada momento. Tras las instituciones psiquiátricas promotoras de la laborterapia, se impone el 
modelo de atención basado en la asistencia en la comunidad que propicia acciones de facilitación laboral en entornos comunitarios como el SEA y el EP. La integración laboral en entornos normalizados y apoyos en el puesto de trabajo caracteriza el SEA; el EP conlleva acciones de inserción laboral que propicien empleo normalizado. Es escasa implantación del SEA en España con personas con TMS, colectivo con el que es más frecuente en nuestro país el uso de EP.

Muchos estudios coinciden en señalar la importancia de determinar las variables predictoras de las intervenciones asistenciales, para favorecer su eficiente aplicación. Numerosos trabajos de investigación se han realizado para estudiar de variables indicativas de éxito o fracaso de la rehabilitación laboral con personas con TMS; la heterogeneidad de las poblaciones, los diferentes programas de rehabilitación laboral, las variadas metodologias de investigación, de medición de variables y de evaluación de resultados, está haciendo inviable sacar conclusiones definitivas. De tales estudios se pueden señalar ciertos resultados, que por su consistencia, pueden tenerse en cuenta:

- La formación y experiencia laboral previa correlaciona positivamente con el desempeño posterior.

- Tener deseos de trabajar favorece la realización de actividad laboral.

- Sintomatología negativa y déficit de habilidades sociales son factores entorpecedores.

- Dentro de los distintos diagnósticos que conllevan los TMS, es la esquizofrenia quién se asocia a mayor déficit de actividad laboral.

- El nivel de las funciones cognitivas es predictor del mantenimiento del empleo.

- Respecto de la relación de las variables sociodemográficas y del tratamiento farmacológico con la actividad laboral, no hay datos concluyentes.

- SEA es un predictor positivo de desempeño laboral.

Se está realizando un enorme esfuerzo científico no habiendo dado aún el fruto deseado, pues no disponemos de evidencia científica inequivoca de las variables que influyen sobre el acceso y mantenimiento del empleo de personas con TMS. Las variables, que los estudios indicados han constatado que tienen capacidad predictora, no necesariamente lo tienen o lo tienen de igual manera con población de este colectivo de nacionalidad española, ya que sobre todo las variables psicosociales pueden tener una influencia diferente con grupos poblacionales diferentes; se precisa por tanto disponer de investigación sobre variables predictoras de rehabilitación laboral de españoles con TMS. 


\section{BIBLIOGRAFÍA}

Aguado, A. L. (1995). Historia de las deficiencias. Madrid: Escuela Libre Editorial. Fundación ONCE. Colección Tesis y Praxis.

Aldaz, J. A. y Vázquez, C. (1996). Esquizofrenia: fundamentos psicológicos y psiquiátricos de la rehabilitación. Madrid: Editorial Siglo XXI.

Ancil, R. J., Holliday, S. y Higgenbttam, J. (1994). Schizophrenia; exploring the spectrum of psychosis. Chichester: Wiley and Sons.

Andia, A. M., Zisook, S., Heaton, R. K., Hesselink, J. y Jernigan, T. (1995). Gender Differences in Schizophrenia. Journal of Nervous and Mental Disease, 183, (18), 522-527.

Angermeyer, M. C., Kühn, L. y Goldstein, J. M. (1990). Gender and the Course of Schizophrenia. Differences in Treated Outcomes. Schizophrenia Bulletin, 16, (2), 293-306.

Anthony, A. W. (1994). Characteristics of people with psychiatric disabilities that are predictive of entry into the rehabilitation process and successful employement. Psychosocial Rehabilitation Journal, 17, (3): 3-13.

Anthony, W. A. y Blanch, A. (1987). Supported employment for persons who are psychiatrically disabled: an historical and conceptual perspective. Psychosocial Rehabilitation Journal, 11, 5-23.

Anthony, W. A., Cohen, M. R. y Farkas, M. D. (1990). Psychiatric rehabilitation. Boston, MA: Boston University, Center for Psychiatric Rehabilitation.

Anthony, W. A., Rogers, E. S., Cohen, M. y Davies, R. R. (1995). Relationships between psychiatric symptomatology, work skills, and future vocational performance. Psychiatric Services, 46, 353-358.

Asociación Americana de Psiquiatría (1995). Manual diagnóstico y estadístico de los trastornos mentales (DSM-IV). Barcelona: Ed. Masson.

Asociación Americana de Psiquiatría (2003). Manual diagnóstico y estadístico de los trastornos mentales (DSM-IV-TR). Barcelona: Ed. Masson.

Bailer, J., Bräuer, W. y Rey, E. R. (1996). Premorbid adjustment as predictor of outcome in schizophrenia: results of a prospective study. Acta Psychiatrica Scandinavica, 93, 368-377.

Banks, B., Charleston, S., Grossi, T. y Mank, D. (2001). Workplace supports, job performance, and integration outcomes for people with psychiatric disabilities. Psychiatric Rehabilitation Journal, 24, (4), 389-396.

Bebout, R. R. y Harris, M. (1995). Personal myths about work and mental illness: response to Lysaker and Bell. Psychiatry Journal, 58, 401-404.

Becker, D. R., Drake, R. E., Bond, G. R., Xie, H., Dain, B. J. y Harrison, K. (1998). Job terminations among persons with severe mental illness participating in supported employment. Community Mental Health Journal, 34, (1).

Becker, D. R. y Smith, J. (2001). Fidelity of Supported Employment Programs and employment outcomes. Psychiatric Services, 52, (6), 834-836.

Bell, M. D. y Bryson, G. (2001). Work rehabilitation in Schizophrenia: Does cognitive impairment limit improvement?. Schizophrenia Bulletin, 27, (2), 269-279.

Bell, M., Bryson, G., Greig, T., Corcoran, C. y Wexler, B. E. (2001). Neurocognitive enhancement therapy with work therapy: effects on neuropsychological test performance. Archives of General Psychiatry, 58, 763-768.

Bell, M. D., Lysaker, P. H. y Milstein, R. M. (1996). Clinical benfits of paid work activity in Schizophrenia. Schizophrenia Bulletin, 22, (1), 5167.

Bellack, A. S., Gold, J. M. y Buchanan, R. W. (1999). Cognitive rehabilitation for schizophrenia: problems, prospects, and strategies. Schizophrenia Bulletin, 25, 257-274.

Boardman, J., Grove, B., Perkins R. y Shepherd, G. (2003). Work and employment for people with psychiatric disabilities. British Journal of Psychiatry, 182, 467-468.

Bond, G. R. (1992). Vocational rehabilitation. En Liberman, R. P. (Eds.). Handbook of Psychiatric Rehabilitation (244-275). New York: Macmillan.

Bond, G. R. (1994). Applying Psychiatric Rehabilitation principles to employment: recent findings. 
En Ancil, R. J., Holliday, S. y Higgenbttam, J. (Eds.). Schizophrenia; exploring the spectrum of psychosis (49-65). Chichester: Wiley and Sons.

Bond, G. R. (1998). Principles of the Individual Placement and Support model: empirical support. Psychiatric Rehabilitation Journal, 22, 11-23.

Bond, G. R. (2003). La integración laboral de personas con trastorno mental severo en Estados Unidos. En Lara, L. y López, M. (Eds.): Integración laboral de personas con trastorno mental severo (103-114). Sevilla: FAISEM.

Bond, G. R., Becker, D. R., Drake, R. E., Rapp, C. A., Meisler, N. y Lehman, A. F. (2001). Implementing supported employment as an evidencebased practice. Psychiatric Services, 52, 313-322.

Bond, G. R., Dietzen, L., McGrew, J. y Miller, L. (1995). Accelerating entry into supported employment for persons with severe psychiatric disabilities. Rehabilitation Psychology, 40, 91-111.

Bond, G. R. y Drake, R. E. (2008). Predictors of competitive employment among patients with schizophrenia. Current Opinion in Psychiatry, 21, (4), 362-369.

Bond, G. R., Drake, R. E., Becker, D. R. y Mueser, K. T. (1999). Effectiveness of psychiatric rehabilitation approaches for employment of people with severe mental illness. Journal Disability Policy Studies, 10, 18-52.

Bond, G. R., Drake, R. E., Mueser, K. T. y Becker, D. R. (1997). An update on supported employment for people with severe mental illness. Psychiatric Services, 48, 335-346.

Bond, G. R., Drake, R. E., Mueser, K. T. y Latimer, E. (2001). Assertive community treatment for people with severe mental illness: critical ingredients and impact on clients. Disease Management and Health Outcomes, 9, 141-159.

Bond, G. R., Kim, H. W., Meyer, P. S., Gibson, J., Tunis, S., Evans, J. D., Lysaker, P., McCoy, M. L., Dincin, J. y Xie H. (2004). Response to Vocational Rehabilitation During Treatment With First or Second Generation Antipsychotics. Psychiatric Services, 55, (1), 59-66.

Bond, G. R. y Meyer, P. S. (1999). The role of medications in the employment of people with schizophrenia. Journal Rehabilitation, 65, 9-16.

Brekke, J. S., Levin, S., Wolkon, G. H., Sobel,
E. y Slade, E. (1993). Psychosocial functioning and subjective experience in schizophrenia. Schizophrenia Bulletin, 19, (3), 599-608.

Brekke, J. S., Raine, A., Ansel, M., Lencz, T. y Bird, L. (1997). Neuropsychological and psychophysiological correlates of psychosocial functioning in schizophrenia. Schizophrenia Bulletin, 23, 19-28.

Bryson, G., Bell, M. D., Kaplan, E. y Greig, T. (1998). The functional consequences of memory impairments on initial work performance in people with schizophrenia. Journal of Nervous and Mental Disease, 186, 610-615.

Bryson, G., Lysaker, P. y Bell, M. (2002). Quality of life benefits of paid work activity in Schizophrenia. Schizophrenia Bulletin, 28, (2), 249-257.

Burns, T., Catty, J., White, S., Becker, T., Koletsi, M., Fioritti, A., Rössler, W., Tomov, T., Van Busschbach, J., Wiersma, D. y Lauber, C. (2008). The Impact of Supported Employment and Working on Clinical and Social Functioning: Results of an International Study of Individual Placement and Support. Schizophrenia Bulletin, 21.

Bustillo, J.; Lauriello, J.; Horan, W. y Keith, S. (2001). The psychosocial treatment of schizophrenia: an update. American Journal of Psychiatry, 158, (2), 163-175.

Caldwell, C. B. y Gottesman, I. I. (1990). Schizophrenics kill themselves too: a review of risk factors for suicide. Schizophrenia Bulletin, 16, 571589.

Caleidoscopia Investigación Social, S. L. (1997). Factores personales y sociales de la integración laboral de las personas con discapacidad. Madrid: Documentos del Real Patronato de Prevención y de Atención a Personas con Minusvalía.

Carpenter, W. T. y Keith, S. J. (1986). Integrating Treatments in Schizophrenia. Psychiatric Clinic North American, 9, 153-164.

Casper, E. S. y Fishbein, S. (2000). Job satisfaction and job success as moderators of the selfesteem of people with mental illness. Psychiatric Rehabilitation Journal, 26, (3), 33-42.

Catty, J., Lissouba, P., White, S., Becker, T., Drake, R. E., Fioritti, A., Knapp, M., Lauber, C., Rössler, W., Tomov, T., Van Busschbach, J., Wiersma, D. y Burns, T. (2008). Predictors of employment for people with severe mental illness: results 
of an international six-centre randomised controlled trial. British Journal of Psychiatry, 192, (3), 224-231.

Ciardiello, J. A. y Bell, M. D. (1988). Vocational rehabilitation of person with ptolongued psychiatric disorders. Baltimore: Johns Hopkins University Press.

Collins, P. Y., Adler, F. W., Boero, M. y Susser, E. (1999). Using local resources in Patagonia: primary care and mental health in Neuquen, Argentina. International Journal of Mental Health, 28, 316.

Collins, P. Y., Lumerman, J., Conover, S. y Susser, E. (1999). Using local resources in Patagonia: a model of community-based rehabilitation. International Journal of Mental Health, 28, 17-24.

Cook, J. A., Lehman, A. F., Drake, R., McFarlane, W. R., Gold, P. B., Leff, H. S., Blyler, C. R., Toprac, M. G., Razzano, L. A., Burke-Miller, J. K., Blankertz, L. E., Shafer, M. S., Pickett-Schenk, S. A. y Grey, D. D. (2005). Integration of psychiatric and vocational services: a multisite randomized, controlled trial of supported employment. American Journal of Psychiatry, 162, (10), 1948-1956.

Cook, J. A. y Razzano, L. (2000). Vocational rehabilitation for persons with schizophrenia: research and implications for practice. Schizophrenia Bulletin, 26, (1), 87-103.

Crowther, R. E., Marshall, M., Bond, G. R. y Huxley, P. (2001). Helping people with severe mental illness to obtain work: systematic review. British Medical Journal, 322, 204-208.

Cunningham, K., Wolbert, R. y Brockmeier, M. B. (2000). Moving beyond the illness: factors contributing to gaining and maintaining employment. American Journal of Community Psychology, 28, (4), 481-494.

Danley, K. S. (1993). Supoorted employment for people with psychiatric disabilities. Lisboa: Conferencia Internacional sobre el Empleo con Apoyo en un Medio Laboral Competitivo.

Daradkeh, T. K. y Karim, L. (1994). Predictors of employment status of treated patients with DSM-III-R diagnoses: can logistic regresión model find a solution?. International Journal of Social Psychiatry, 40, 141-149.

Davis, L. W., Lysaker, P. H., Lancaster, R. S., Bryson, G. J. y Bell, M. D. (2005). The Indianapolis
Vocational Intervention Program: a cognitive behavioral approach to addressing rehabilitation issues in schizophrenia. Journal of Rehabilitatioon Research and Development, 42, (1), 35-46.

Davis, L. W., Nees, M. A., Hunter, N. L. y Lysaker, P. H. (2004). Hopelessness as a predictor of work functioning among patients with schizophrenia. Psychiatric Services, 55, (4).

De Giacomo, P., Pierri, G., Rugiu, A. S., Buonsante, M., Vadruccio, F. y Zavoianni, L. (1997). Schizophrenia: a study comparing a family therapy group following a paradoxical model plus psychodrugs and a group treated by the conventional clinical approach. Acta Psychiatrica Scandinavica, 95, (3), 183-188.

Defensor del Pueblo (1992). Situación jurídica y asistencial del enfermo mental en España. Madrid: Oficina del Defensor del Pueblo.

Desviat, M (1995). La reforma psiquiátrica. Madrid: Dors.

Dickinson, D., Bellack, A. S. y Gold, J. M. (2006). Social/Communication skills, cognition, and vocational functioning in schizophrenia. Schizophrenia Bulletin, 33, 1213-1220

Dilk, M. N. y Bond, G. R. (1996). Meta-analytic evaluation of skills training research for individuals with severe mental illness. Journal of Cosulting and Clinical Psychology, 64: 1337-1346.

Dixon, L., Adams, C. y Lucksted, A. (2000). Update on family psycho-education for schizophrenia. Schizophrenia Bulletin, 26, 5-20.

Dorio, J., Guitar, A., Solheim, L., Dvorkin, C. y Marine, S. (2002). Differences in job retention in a supported employment program Chinook Clubhouse. Psychiatric Rehabilitation Journal, 25, (3), 289-298.

Drake, R. E., McHugo, G. J., Bebout, R. R., Becker, D. R., Harris, M., Bond, G. R., y Quimby, E. (1999). A randomized clinical trial of supported employment for inner-city patients with severe mental illness. Archives of General Psychiatry, 56, 627-633.

Drake, R. E., McHugo, G. J., Becker, D. R., Anthony, W. A. y Clark, R. E. (1996). The New Hampshire study of supported employment for people with severe mental illness: vocational outcomes. Journal of Consulting and Clinical Psychology, 64, 391-399. 
Evans, J. D., Bond, G. R., Meyer, P. S., Kim, H. W., Lysaker, P. H., Gibson, P. J. y Tunis, S. L. (2004). Cognitive and clinical predictors of success in vocational rehabilitation in schizophrenia. Schizophrenia Research , 70, (2-3), 331-42.

Farina, A. (1998). Stigma. En Mueser, K. T. y Tarrier, N. (Eds.): Handbook of social functioning in schizophrenia (247-279). Boston: Allyn y Bacon.

FEAFES (1995). Estudio europeo sobre las necesidades de los familiares de enfermos mentales. EUFAMI (trabajo no publicado).

Furlong, M., Mccoy, M. L., Dincin, J., Clay, R., McClory, K. y Pavick, D. (2002). Jobs for people with the most severe psychiatric disorders; thresholds bridge north pilot. Psychiatric Rehabilitation Journal, 26, (1), 13-22.

Galvis, C. R.. Pinzón, M. L. y Romero, E. (2004). Comparación entre la habilidad de cuidado de cuidadores de personas en situación de enfermedad crónica en Villavicencio Meta. Avances en Enfermería, 22, (1), 4-26.

Gallio, G. (1987). Nell'Impresa Sociale. Trieste: Per la Salute Mentale.

Garety, P., Fowler, D., Kuipers, E., Dunn, G., Bebbington, P., Freeman, D., Hadley, C. (1997). London-East Anglia randomised controlled trial of cognitive-behavioural therapy for psychosis: II, predictors of outcome. Journal of Psychiatry, 171, 420-426.

Gervey, R. y Bedell, J. R. (1993). Supported employment, in Psychological Assessments and Treatment of Persons With Severe Mental Disorders. Washington, DC: Bedell JR.

Gisbert, C. (2002). Rehabilitación psicosocial del trastorno mental severo; situación actual y recomendaciones. Madrid: Asociación Española de Neuropsiquiatría.

Goergen, R. (2003). Empresas y empresarios sociales en Italia: la experiencia de Trieste. En Lara, L. y López, M. (2003): Integración laboral de personas con trastorno mental severo (158-200). Sevilla: FAISEM.

Gold, J. M., Goldberg, R. W., McNary, S. W., Dixon, L. B. y Lehman, A. F. (2002). Cognitive Correlates of Job Tenure Among With Severe Mental Illness. American Journal of Psychiatry, 159, 13951402.

Gold, J. M., Queern, C., Iannone, V. N. y
Buchanan, R. W. (1999). Repeatable Battery for the Assessment of Neuropsychological Status as a screening test in schizophrenia, I: sensitivity, reliability, and validity. American Journal of Psychiatry, 156, 1937-1950.

Goldberg, R. W., Lucksted, A., McNary, S., Gold, J. M., Dixon, L. y Lehman, A. (2001). Correlates of long-term unemployment among inner-city adults with serious and persistent mental illness. Psychiatric Services, 52, 101-103.

Gould, R. A., Mueser, K. T., Bolton, E., Mays, V. y Goff, D. (2001). Cognitive therapy for psychosis in schizophrenia: a preliminary meta-analysis. Schizophrenia Research, 48, 335-342.

Gowdy, E.L.; Carlson, L.S. y Rapp, C. A. (2003). Practices differentiating high-performing from lowperforming supported employment programs. Psychiatric Rehabilitation Journal, 26, (3), 232-239.

Green, M. F., Kern, R. S., Braff, D. L. y Mintz, J. (2000). Neurocognitive deficits and functional outcome in schizophrenia: Are we measuring the "right stuff"?. Schizophrenia Bulletin, 26, (1), 119136.

Green, M. F., Kern, R. S. y Sergi, M. J. (1999). An introduction to neurocognitive dysfunction in schizophrenia: Why should we care?. En Keefe, R. S. (1999). Improving Cognitive Function in the Schizophrenic Patient (1-11). London: Science Press.

Grove, B., Freudenberg, M., Harding, A. y O'Flynn, D. (1997). The social firm handbook. Brighton: Pavilion.

Gureje, O., Odejide, A. O., Olatawura, M. O., Ikuesan, B. A., Acha, R. A., Bamidele, R. W. y Raji, O. S. (1995). Results from the Ibadan centre. En Ustun T. B. y Sartorius, N. (Ed.): Mental illness in general health care: an international study (157173). Chichester: John Wiley y Sons on behalf of the World Health Organization.

Hadley, T. R., Turk, R. y McGurrin, M. (1997). Community treatment teams: an alternative to state hospitals. Psychiatric Quarterly, 68, 77-90.

Hatfield, A. B. y Lefley, H. P. (1993). Surviving Mental Illness: Stress, Coping, and Adaptation. New York: Guilford.

Henry, A. D., Barreira, P., Banks, S., Brown, J. M. y McKay, C. (2001). A retrospective study of Clubhouse-based transitional employment. Psychiatric Rehabilitation Journal, 24, (4), 344-354. 
Hoffmann, H. y Kupper, Z. (1996). Patient dynamics in early stages of vocational rehabilitation: a pilot study. Comprehensive Psychiatry, 37, (3), 216-221.

Hoffman, H. y Kupper, Z. (1997). Relationships between social competence, psychopathology and work performance and their predictive value for vocational rehabilitation of schizophrenic outpatients. Schizophrenia Research, 23, 69-79.

Hogarty, G. E., Kornblith, S. J., Greenwald, D., DiBarry, A. L., Cooley, S., Ulrich, R. F., Carter, M. y Flesher, S. (1997). Three-year trial of personal therapy among schizophrenic patients living with or independent of family. I: description of study and effects of relapse rates. American Journal of Psychiatry, 154, (11), 1504-1515.

Holthausen, E. A., Wiersma, D., Cahn, W., Kahn, R. S., Dingemans, P. M., Schene, A. H. y Van den Bosch, R. J. (2007). Predictive value of cognition for different domains of outcome in recent-onset schizophrenia. Psychiatry Research, 149, 71-80.

Holzner, B., Kemmler, G. y Meise, U. (1998). The impact of work-related rehabilitation on the quality of life of patients with Schizophrenia. Social Psychiatry and Psychiatric Epidemiology, 33, 624-631.

Hume, C. y Pullen, I. (1995). Rehabilitation in psychiatry. Edinburgh: Ed. Churchill Livingstone.

IMSERSO (2007a). Modelo de atención a las personas con enfermedad mental grave. Madrid: $\mathrm{M}^{\mathrm{0}} \mathrm{de}$ Trabajo y Asuntos Sociales.

IMSERSO (2007b). Modelo de Centro de Rehabilitación Psicosocial. Madrid: $\mathrm{M}^{\mathrm{0}}$ de Educación, Política Social y Deporte.

Jaeger, J. y Douglas, E. (1992). Neuropsychiatric rehabilitation for persistent mental illness. Psychiatric Quarterly, 63, 71-94.

James, D. (1983). The experimental treatment of two cases of auditory hallucinations. British Journal of Psychiatry, 143, 515-526.

Jeffery, D. P., Ley, A., McLaren, S. y Siegfried, N. (2002). Programas terapéuticos psicosociales para personas tanto con enfermedades mentales graves como por abuso de sustancias. En Cochrane Plus: Biblioteca Cochrane Plus. Oxford: Update Software Ltd. (2006).

Jones, C. J., Cormac, I., Silveira da Mota J. I. y
Campbell, C. (2004). Terapia cognitivo-conductual para la esquizofrenia. En Cochrane Plus: Biblioteca Cochrane Plus. Oxford: Update Software Ltd. (2006).

Jones, C. J., Perkins, D. y Born, D. L. (2001). Predicting work outcomes and service use in supported employment services for persons with psychiatric disabilities. Psychiatric Rehabilitation Journal, 25, (1), 53-59.

Kern, R. S.; Green, M. F. y Satz, P. (1992): Neuropsychological predictors of skills training for chronic psychiatric patients. Psychiatry Research, 43, 223-230.

Kern, R. S., Liberman, R. P., Kopelowicz, A., Mintz, J. y Green, M. F. (2002). Applications of errorless learning for improving work performance in persons with schizophrenia. American Journal of Psychiatry, 159, 1921-1926.

Krabbendam, L. y Aleman, A. (2003). Cognitive rehabilitation in schizophrenia: a quantitative analysis of controlled studies. Psychopharmacology, 169, 376-382.

Kravetz, S., Dellario, D., Granger, B. y Salzer, M. (2003). A two-faceted work participation approach to employment and career development as applied to persons with a psychiatric disability. Psychiatric Rehabilitation Journal , 26, (3), 278-89.

Kulhara, P. y Wig, N. N. (1978). The chronicity of schizophrenia in North-West India. Results of a follow-up study. British Journal of Psychiatry, 132, 186-190.

Lal, S. y Mercier, C. (2002). Thinking out of the box: an intersectorial model for vocational rehabilitation. Psychiatric Rehabilitation Journal, 26, (2), 145-53.

Laloma, M. (2007). Empleo protegido en España. Análisis de la normativa legal y logros alcanzados. Madrid: Ediciones Cinca.

Latimer, E. (1999). Economic impacts of assertive community treatment: a review of the literature. Canadian Journal Psychiatry, 44, 443-454.

Lauriello J., Lenroot, R. y Bustillo, J. R. (2003). Maximizing the sinergy between pharmacotherapy and psychosocial therapies for schizophrenia. Psychiatric Clinic North American, 26: 191-211.

Lebell, M., Marder, R. E., Mintz, J., Mintz, I. L., Thompson, M., Wshing, W., Johnston-Cronk, K. y 
McKenzie, J. (1993). Patines, perceptions family emocional climate and outcome in schizophrenia. British Journal of Psychiatry, 162, 751-754.

Leff, J. y Gamble, C. (1995). Training of community psychiatric nurses in family work for schizophrenia. International Journal of Mental Health, $24,76-88$

Lehman, A. F. (1995). Vocational Rehabilitation in schizophrenia. Schizophrenia Bulletin, 21, (4), 645-656.

Lehman, A. F., Goldberg, R., Dixon, L. B., McNary, S., Postrado, L., Hackman, A. y McDonnell, K. (2002). Improving employment outcomes for persons with severe mental illnesses. Archives of General Psychiatry, 59, 165-172.

Levine, Z., Stahl, B. S., Ruderman, O. S., Babushkin, Y. O. y Bersudsky, R. B. (2006). Homocysteine-reducing strategies improve symptoms in chronic schizophrenic patients with hyperhomocysteinemia. Biological Psychiatry, 60, (3), 265-269.

Liberman, R. P. (1993): Rehabilitación integral del enfermo mental crónico. Barcelona: Ed. Martinez Roca.

Link, B. G., Cullen, F. T., Mirotznik, J. y Struening, E. (1992). Consequences of stigma for persons with mental illness: Evidence from social sciences. En Fink, P. J. y Tasman, A. (Ed.): Stigma and mental illness (87-96). Washington: American Psychiatric Press.

López, M., Alvarez, F. y Laviana, M. (1998). Empresas sociales y rehabilitación psiquiátrica en Andalucía. Revista de la Asociación Española de Neuropsiquiatría, 18, (68), 735-747.

Lysaker, P. H. y Bell, M. D. (1995). Work rehabilitation and improvements in insight in schizophrenia. Journal of Nervous and Mental Disease, 183, 103-106.

Lysaker, P. H., Bell, M. D., Milstein, R. M., Goulet, J. G. y Bryson, G. J. (1993). Work capacity in Schizophrenia. Hospital and Community Psychiatry, 44, 278-280.

Lysaker, P. H., Bell, M. D., Zito, W. S. y Bioty, S. M. (1995). Social skills at work: deficits and predictors of improvement in schizophrenia. Journal of Nervous and Mental Disease, 183, (11), 688-692

Manning, C. y Withe, P. D. (1995). Attitudes of employers to the mentally ill. Psychiatric Bulletin, 19, 541-543.

Marrone, J., Balzell, A. y Gold, M. (1995). Employment supports for people with Mental Illness. Psychiatric Services, 46, (7), 707-711.

Massel, H. K., Corrigan, P. W., Liberman, R. P. y Milan, M. A. (1991). Conversation skills training of thought-disordered schizophrenic patients through attention focussing. Psychiatric Research, 38, 51-61.

Mausbach, B. T., Coon, D. W., Depp, C., Rabinowitz, Y. G., Wilson, E., Thompson, L. W. y Gallagher-Thompson, D. (2004). Ethnicity and time to institutionalization of dementia patients: a comparison of latina and caucasian female family caregivers. Journal of the American Geriatrics Society, 52, (7), 1077-1084.

McDonald-Wilson, K. L., Rogers, S., Massaro, J. M., Lyass, A. y Crean, T. (2002). An investigation of reasonable workplace accommodations for people with psychiatric disabilities: quantitative findings from a multi-site study. Community Mental Health Journal, 38, (1), 35-50.

McFarlane, W. R., Dushay, R. A. Deakins, S. M., Stastny, P., Lukens, E., Toran, J. y Link, B. (2000). Employment outcomes in family-aided assertive community treatment. American Journal Orthopsychiatry, 70, 203-214.

McGlashan, T. H. (1994). Psychosocial treatments of schizophrenia. En Andreasen, C. (Ed.): Schizophrenia from mind to molecule. American psychopathological series (189-215). Washington: American Psychiatric Press.

McGurk, S. R. y Meltzer, H. Y. (2000). The role of cognition in vocational functioning in schizophrenia. Schizophrenia Research, 45, 175-184.

McGurk, S. R. y Mueser, K. T. (2006). Cognitive and clinical predictors of work outcomes in clients with schizophrenia receiving supported employment services: 4-year Follow-Up. Administration and Policy in Mental Health, 5, 598-606.

McGurk, S. R., Mueser, K. T., Harvey, P. D., LaPuglia, R. y Marder, J. (2003). Cognitive and symptom predictors of work outcomes for clients with schizophrenia in supported employment. Psychiatric Services, 54. (8), 1129-1135.

Merinder, L. B., Viuff, A. G., Laugesen, H. D., Clemmensen, K., Misfelt, S. y Espensen, B. (1999). 
Patient and relative education in community psychiatry: a randomized controlled trial regarding its effectiveness. Social Psychiatry and Psychiatric Epidemiology, 34, (6), 287-294.

Meyer, P. S., Bond, G. R., Tunis, S. L. y McCoy, M. L. (2002). Comparison between the effects of atypical and traditional antipsychotics on work status for clients in a psychiatric rehabilitation program. Journal of Clinical Psychiatry, 63, 108116.

Milev, P., Ho, B. C., Arndt, S. y Andreasen, N. C. (2005). Predictive values of neurocognition and negative symptoms on functional outcome in schizophrenia: a longitudinal first-episode study with 7year follow-up. American Journal of Psychiatry, 162, 495-506.

Morrison, R. L. y Bellack, A. S. (1987). Social functioning of schizophrenic patients: clinical and research issues. Schizophrenia Bulletin, 13, (4), 715-725.

Mueser, K. T., Becker, D. R., Torrey, W. C., Xie, H., Bond, G. R., Drake, R. E. y Dain, B. J. (1997). Work and nonvocational domains of functioning in persons with severe mental illness: a longitudinal analysis. Journal of Nervous and Mental Disease, 185, (7), 419-426.

Mueser, K. T., Becker, D. y Wolfe, R. (2001). Supported employment, job preferences, job tenure and satisfaction. Journal of Mental Health, 10, (4), 411-417.

Mueser, K. T., Bond, G. R. y Drake, R. E. (2001). Community-based treatment of schizophrenia and other severe mental disorders: treatment outcomes. Medscape Mental Health (online journal), 6.

Mueser, K. T., Salyers, M. P. y Mueser, P. R. (2001). A prospective analysis of work in Schizophrenia. Schizophrenia Bulletin, 27, (2), 282-296.

Mueser, K. T. y Tarrier, N. (1998). Handbook of social functioning in Schizophrenia. Boston: Allyn y Bacon.

Muntaner, C. y O'Campo, P. (1993). A critical appraisal of the Demand. Control model of the psychosocial work environment: epistemological, social, behavioral and class considerations. Société des Sciences Medicales, 36, 509-517.

Nahikian, M. (1997). Influential factors of caregiver behavior at mealtime: a study of twenty-four day care programs. Journal of the America Dietetic, 97, (5), 505-509.

Nordt, C., Müller, B., Rössler, W. y Lauber, C. (2007). Predictors and course of vocational status, income, and quality of life in people with severe mental illness: a naturalistic study. Social Science y Medicine, 65, (7), 1420-1429.

Nuechterlein, K. H., Dawson, M., Glitin, M. (1992). Developmental processes in schizophrenia disorders: longitudinal studies of vulnerability and stress. Schizophrenia Bulletin, 18, 387-418.

Obiols, J. E. y Vicens-Vilanova, J. (2003). Etiología y signos de riesgo en la esquizofrenia. International Journal of Psychology and Psychological Therapy, 3, (2), 235-250.

O'Flynn, D. y Craig, T. (2001). Which way to work?. Occupations, vocations and opportunities for mental health service users. Journal of Mental Health, 10, (1), 1-4.

Olbrich, R. y Mussgay, L. (1990). Reducton of schizophrenic deficits by cognitive training: an evaluative study. European Archives of Psychiatry and Neurological Sciences, 239, 366-9.

Orviz, S. y González, J. (1994). Factores que correlacionan con incorporación y mantenimiento laboral en personas con problemas psiquiátricos crónicos. Revisión bibliográfica. En Asociación Madrileña de Rehabilitación Psicosocial: II Jornadas de la Asociación Madrileña de Rehabilitación Psicosocial: Tiempo de rehabilitación. Madrid: Asociación Madrileña de Rehabilitación Psicosocial.

Peckham, J. y Muller, J. (1999). Employment and schizophrenia: recommendations to improve employability for individuals with schizophrenia. Psychiatric Rehabilitation Journal, 22, (4), 399-402.

Pekkala, E. y Merinder, L. (2002). Psicoeducación para la esquizofrenia. En Cochrane Plus: Biblioteca Cochrane Plus. Oxford: Update Software Ltd. (2002).

Penn, D. L. y Mueser, K. T. (1996). Research update on the psychosocial treatment of schizophrenia. American Journal of Psychiatry, 153, 607617.

Perkins, R. y Rinaldi, M. (2002). Unemployment rates among patients with long-term mental health problems. A decade of rising unemployment. Psychiatric Bulletin, 26, 295-298. 
Phillips, S. D., Burns, B. J., Edgar, E. R., Mueser, K. T., Linkins, K.W., Rosenheck, R. A. Drake, R. E. y McDonel-Herr, E.C. (2001). Moving assertive community treatment into standard practice. Psychiatric Services, 52, 771-779.

Posininsky, H. y Schaumburg, C. (1996). Schizophrenie was ist das?. Göttingen Zurich: Vandenhoeck y Ruprecht.

Prochaska, J. O., DiClemente, C. C. y Norcross, J. C. (1992). In search of how people change: applications to addictive behaviors. American Psychologist, 47, 1102-1114.

Provencher, H. L., Gregg, R., Mead, S. y Mueser, K. T. (2002). The role of work in the recovery of persons with psychiatric disabilities. Psychiatric Rehabilitation Journal, 26, 2, 132-44.

Racenstein, J. M., Harrow, M., Reed, R., Martin E., Herbener E. y Penn, D. L. (2002). The relationship between positive symptoms and instrumental work functioning in schizophrenia: a 10-year follow-up study. Schizophrenia Research, 56, 95-103.

Razzano, L. A., Cook, J. A., Burke-Miller, J. K., Mueser, K. T., Pickett-Schenk, S. A., Grey, D. D., Goldberg, R. W., Blyler, C. R., Gold, P. B., Leff, H. S., Lehman, H. F., Shafer, M. S., Blankertz, L. E., McFarlane, W. R., Toprac, M. G. y Carey, M. A. (2005). Clinical factors associated with employment among people with severe mental illness: findings from the employment intervention demonstration program. Journal of Nervous and Mental Disease, 193, (11), 705-713.

Resnick, S. G. y Bond, G. (2001). The Indiana Job Satisfaction Scale: job satisfaction in vocational rehabilitation for people with severe mental illness. Psychiatric Rehabilitation Journal, 25, (1), 12-19.

Rogers, E. S., Anthony, W. A., Cohen, M. y Davies, R. R. (1997). Prediction of vocational outcome based on clinical and demographic indicators among vocationally ready clients. Community Mental Health Journal, 33, (2), 99-112.

Rosen, A. y Barfoot, K. (2001): Day care and occupation: Structured rehabilitation and recovery programmes and work. Textbook of Community Psychiatry (295-308). Oxford: Oxford University Press.

Rosenheck, R. A., Leslie, D., Keefe, R., McEvoy, J., Swartz, M., Perkins, D., Stroup, S., Hsiao, J. K. y Lieberman, J. (2006). Barriers to employment for people with schizophrenia. American Journal of Psychiatry, 163, 411-417.
Rosenheck, R. A., Neale, M. S., Leaf, P., Milstein, R. y Frisman, L. (1995). Multisite experimental cost study of intensive psychiatric community care. Schizophrenia Bulletin, 21, 129-140.

Salkever, D. S., Karakus, M. C., Slade, E. P., Harding, C. M., Hough, R. L., Rosenheck, R. A., Swartz, M. S., Barrio, C. y Yamada, A. M. (2007). Measures and Predictors of Community-Based Employment and Earnings of Persons With schizophrenia in a Multisite Study. Psychiatric Services, 58, (3).

Saraceno, B. (1995). La fine dell'Intrattenimento. Milán: Etas Libri.

Sartorius, N. (1997). Fighting schizophrenia and its stigma. A new World Psychiatric Association educational programme. British Journal of Psychiatry, 170, 297.

Schwarz, G. (2003). Las empresas sociales como medio para la integración laboral de personas con trastorno mental severo en Alemania. En Lara, L. y López, M. (Eds.): Integración laboral de personas con trastorno mental severo (150-157). Sevilla: FAISEM.

Sergi, M. J., Kern, R. S., Mintz, J. y Green M. F. (2005). Learning potential and the prediction of work skill acquisition in schizophrenia. Schizophrenia Bulletin, 31, (1), 67-72.

Sharma, T. y Harvey, P. D. (2000). Cognitive enhancement as a treatment strategy in schizophrenia. En Sharma, T. y Harvey, P. (Eds.): Cognition in Schizophrenia. Impairments, Importance and Treatment Strategies (286-302). Oxford: Oxford University Press.

Shepherd, G. (1989). The value of work in the 1980's. Psychiatric Bulletin, 13, 231-233.

Shepherd, G. (2003). Integración laboral de personas con enfermedad mental severa. Perspectiva nacional y local. En Lara, L. y López, M. (2003). Integración laboral de personas con trastorno mental severo. Sevilla: FAISEM.

Shepherd M., Watt, D., Falloon I. y Smeeton, N. (1989). The natural history of schizophrenia: a fiveyear follow-up study of outcome and prediction in a representative sample of schizophrenics. Psycology Medicine, 19, (15), 1-46.

Simmons, T. J., Selleck, L., Steele, R. B. y Sepetauc, F. (1993). Supports and rehabilitation for employment. En Flexer, R. W. y Solomon, P. L. 
(Eds.): Psychiatric Rehabilitation in practice. Boston: Ed. Andover.

Slade, E. y Salkever, D. (2001). Symptom effects on employment in a structural model of mental illness and treatment: analysis of patients with schizophrenia. Journal of Mental Health Policy and Economics, 4, 25-34.

Snell, M. E. (1983). Systematic instruction of the moderately and severely handicapped. Columbus, $\mathrm{OH}$ : Charles E. Merrill.

Sobrino, T. (2003). Centros de rehabilitación laboral. En Instituto Universitario de Integración en la Comunidad: II Congreso Ibérico de Salud Mental: Rehabilitación en Salud Mental. Atención Comunitaria, Rehabilitación y Empleo. Salamanca: Instituto Universitario de Integración en la Comunidad.

Suslow, T., Schonauer, K., Ohrmann, P., Eikelmann, B. y Reker, T. (2000). Prediction of work performance by clinical symptoms and cognitive skills in schizophrenic outpatients. Journal of Nervous and Mental Disease, 188, 116-118.

Talbott, J. A. (1984). The chronic mental patient: a national perspective. En Mirabi M.: The chronically mentally ill: research and services (332). New York: Spectrum Publications.

Tarrier, N. (1987). An investigation of residual psychotic symptoms in discharged schizophrenic patients. British Journal of Clinical Psychology, 26, 141-3.

Tomov, T. (1999). Central and eastern european countries. En. Thornicroft, G. y Tansella, G. (Ed.). The mental health matrix: a manual to improve services (216-227). Cambridge: Cambridge University Press.

Torrey, W. C., Mueser, K. T., McHugo, G. H. y Drake, R. E. (2000). Self-esteem as an outcome measure in studies of vocational rehabilitation for adults with severe mental illness. Psychiatric Services, 51, 229-233.

Turton, N. (2001). Welfare benefits and work disincentives. Journal of Mental Health, 10, (3), 285-300.
Vauth, R., Corrigan, P. W., Clauss, M., Dietl, M., Dreher-Rudolph, M., Stieglitz, R. D. y Vater R. (2005). Cognitive Strategies Versus Self-Management Skills as Adjunct to Vocational Rehabilitation. Schizophrenia Bulletin, 31, (1), 55-66.

Velligan, D. I., Bow-Thomas, C. C., Miller, A. L. y Halgunseth, L. C. (2000). Do specific neurocognitive deficits predict specific domains of community function in Schizophrenia?. The Journal of Nervous and Mental Disease, 188, (8), 518-524.

Verdugo, M. A., Jordán de Urries, F. B. y Bellver, F. (1998). Situación actual del Empleo con Apoyo en España. Siglo Cero, 175, (29-1), 23-31.

Verdugo, M. A. y Jordán de Urries, B. (2003). Empleo con apoyo y salud mental. Ithaca, NY: GLADNET Collection, Cornell University.

Wallace, C. J., Tauber, R. y Wilde, J. (1999). Teaching fundamental workplace skills to persons with Serious mental Illness. Psychiatric Services, 50, (9), 1147-1153.

Warner, R. (2001). Work disincentives in US disability pension programs. Journal of Mental Health, 10, (4), 405-409.

Wehman, P. (1981). Competitive employment: new horizons for severely disabled individuals. Baltimore: Ed. Brookes.

Wehman, P., Moon, S., Everson, J. M., Wood, W. y Barcus, J. J. (1987). Transition from school to work. New challenges for youth with severe disabilities. Baltimore: Paul H. Brookes.

Wiedl, K. H. (1999). Assessing cognitive modifiability as a supplement to readiness for rehabilitation in schizophrenic patients. Psychiatric Services, 50, 1411-1419.

Wing, J. K. (1992). Esquizofrenia. En Watts, F. y Bennet, D. (Eds.). Teoría y práctica de la rehabilitación psiquiátrica (59-77). México: Ed. Limusa.

Wyatt, R. J. (1991). Schizophrenics and the natural course of schizophrenia. Schizophrenia Bulletin, 17, 325-351. 